M. Taroni

C. J. W. Breward

L. J. Cummings

I. M. Griffiths 



\title{
Asymptotic solutions of glass temperature profiles during steady optical fibre drawing
}

\author{
M. Taroni ${ }^{1}$ (taroni@maths.ox.ac.uk), C. J. W. Breward ${ }^{1}$ \\ (breward@maths.ox.ac.uk), L. J. Cummings ${ }^{2}$ \\ (linda.cummings@njit.edu), and I. M. Griffiths ${ }^{1} \dagger$ \\ (griffit4@maths.ox.ac.uk) \\ ${ }^{1}$ Oxford Centre for Collaborative Applied Mathematics, Mathematical Institute, \\ University of Oxford, 24-29 St Giles', Oxford OX1 3LB, UK \\ ${ }^{2}$ Department of Mathematical Sciences, New Jersey Institute of Technology, \\ Newark, N.J. 07102 USA
}

September 28, 2012

\begin{abstract}
.
In this paper we derive realistic simplified models for the high-speed drawing of glass optical fibres via the downdraw method, that capture the fluid dynamics and heat transport in the fibre via conduction, convection and radiative heating. We exploit the small aspect ratio of the fibre and the relative orders of magnitude of the dimensionless parameters that characterize the heat transfer to reduce the problem to one- or two-dimensional systems via asymptotic analysis. The resulting equations may be readily solved numerically and in many cases admit exact analytic solutions. The systematic asymptotic breakdown presented is used to elucidate the relative importance of furnace temperature profile, convection, surface radiation and conduction in each portion of the furnace and the role of each in controlling the glass temperature.

The models derived predict many of the qualitative features observed in the real industrial process, such as the glass temperature profile within the furnace and the sharp transition in fibre thickness. The models thus offer a desirable route to quick scenario testing, providing valuable practical information into the dependencies of the solution on the parameters and the dominant heat-transport mechanism.
\end{abstract}

Keywords: asymptotic analysis, slow viscous flow, heat transfer, extensional flow, optical fibre drawing

\section{Table of Contents}

1 Introduction 2

2 Problem description 4

3 Fluid flow 10

4 Temperature profiles 11

5 Discussion 23

A Phase plane analysis at constant furnace temperature 25

References

\footnotetext{
$\dagger$ Corresponding author.
}

(C) 2012 Kluwer Academic Publishers. Printed in the Netherlands. 


\section{Introduction}

Fibre drawing is an important industrial process used to manufacture fibre-optic cables for telecommunications applications. A cylindrical glass preform, of diameter around 5 centimetres, is lowered into a furnace. Here the preform melts, the glass viscosity decreases, and a fibre of thickness around 100 microns may be drawn by applying a tension at the end. As it exits the furnace, the glass is cooled by the air and solidifies into the desired solid fibre. A schematic of the process is shown in Fig. 1.

One of the most interesting aspects of the process is that it is not isothermal and many of the material properties, most notably the glass viscosity, are found to depend on the temperature. In general, the glass temperature is governed by a balance between thermal convection, radiation, and conduction, although the relative importance of these effects changes with distance down the fibre. In particular, there is typically a sharp transition in the glass thickness, with the glass behaving differently on either side of the transition point.

There have been many theoretical studies of the drawing process in the literature stretching back over 40 years. Early work considered the one-dimensional problem, in which quantities of interest vary only in the axial direction and not radially (Paek and Runk, 1978, Geyling and Homsy, 1980). Later studies concentrated on improving the modelling of the radiative heat transfer (Myers, 1989), with Jaluria and co-workers gradually developing methods to solve the full threedimensional conjugate heat transfer between the glass, surrounding gas, and furnace (Lee and Jaluria, 1997, Roy Choudhury et al., 1999). However, these solutions are computationally very demanding, and so simpler models remain desirable for quick scenario testing and to give clearer insight into the dependencies of the solution on the parameters. To that end, Huang and co-workers extended earlier one-dimensional models to investigate the effects of, for example, viscous dissipation (Wylie and Huang, 2007) and dopant diffusion (Huang et al., 2008), but often at the expense of realistic radiative modelling, which is an inherent part of the industrial process. Other studies ignored radiative heating altogether and considered only the cooling stage of the process (Papamichael and Miaoulis, 1991). Further, the majority of work in this area considers fibre drawing with a draw speed of comparable magnitude with the input speed, while fabrication of thin optical fibres necessitates much higher draw speeds that have become possible more recently (Yin and Jaluria, 2000).

In this paper we derive simplified but realistic models for highspeed fibre drawing for the fabrication of optical fibres, including both 


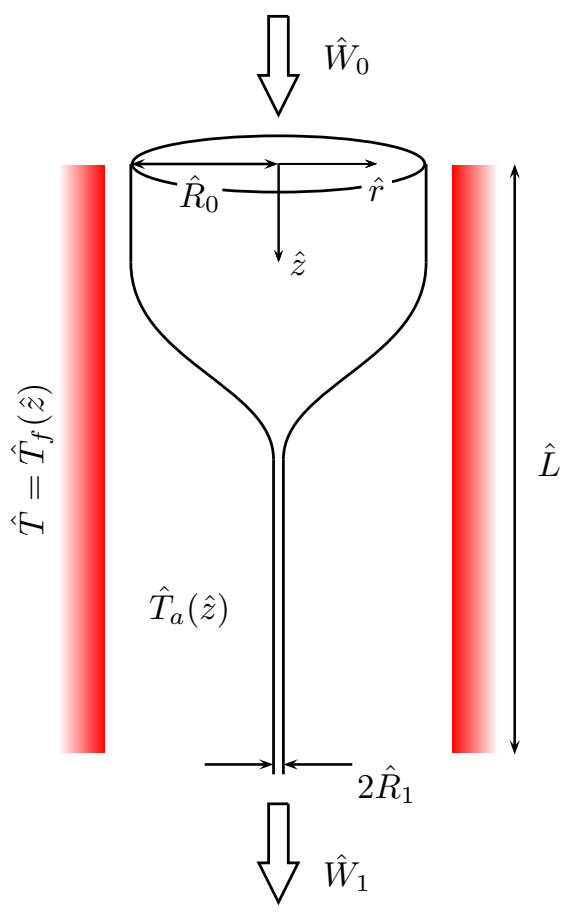

Figure 1. Schematic diagram for the drawing of optical glass fibres. The (dimensional) furnace temperature is given by $\hat{T}_{f}(\hat{z})$ while the ambient air temperature in the furnace is denoted $\hat{T}_{a}(\hat{z})$.

radiative and convective heat transfer throughout the process and determine where each mechanism dominates. We exploit both the small aspect ratio of the fibre and the relative orders of magnitude of the dimensionless parameters to reduce the problem to a one- or twodimensional system of equations that may be readily solved numerically, and for which some approximate analytical solutions exist. In particular, we aim to give a systematic asymptotic breakdown of the dependencies of convection, surface radiation, and conduction, and how their relative importance changes with axial distance down the furnace. We investigate different possible functional forms for the radiusdependent radiative and convective heat transfer coefficients, and in addition consider the effect of non-uniform furnace temperatures.

The paper is laid out as follows. In $\S 2$ we formulate our theoretical description of the problem, coupling together the axisymmetric slowflow equations with an energy equation governing the temperature of the glass. In $\S 3$ we exploit the small aspect ratio of the fibre to derive one-dimensional equations for the fluid flow. We then consider the tem- 
perature profiles of the glass in $\S 4$, considering a number of different asymptotic limits that may be relevant to the industrial process. Finally in $\S 5$ we discuss our results and consider possible avenues for future research.

\section{Problem description}

\subsection{Governing EQUATIOns}

\subsubsection{Fluid flow}

We consider the steady-state configuration where the input radius of the fibre preform is $\hat{R}_{0}$ and the length of the furnace is $\hat{L}$, as depicted in Fig. 1, and we note that for clarity all dimensional quantities are denoted with hats. Based on typical parameter values for the process, given in Table I, we find that the Reynolds number for the flow, based on the fibre radius, is very small and, assuming axisymmetry, the fluid flow is governed by the slow-flow equations (Fitt et al., 2002),

$$
\begin{gathered}
\frac{1}{\hat{r}} \frac{\partial}{\partial \hat{r}}(\hat{r} \hat{u})+\frac{\partial \hat{w}}{\partial \hat{z}}=0 \\
-\frac{\partial \hat{p}}{\partial \hat{r}}+\frac{\partial}{\partial \hat{z}}\left(\hat{\mu} \frac{\partial \hat{u}}{\partial \hat{z}}\right)+\hat{\mu} \frac{\partial}{\partial \hat{r}}\left(\frac{1}{\hat{r}} \frac{\partial}{\partial \hat{r}}\left(\hat{r} \frac{\partial \hat{u}}{\partial \hat{r}}\right)\right)+\frac{\partial \hat{\mu}}{\partial \hat{z}} \frac{\partial \hat{w}}{\partial \hat{r}}+2 \frac{\partial \hat{\mu}}{\partial \hat{r}} \frac{\partial \hat{u}}{\partial \hat{r}}=0 \\
-\frac{\partial \hat{p}}{\partial \hat{z}}+\frac{\partial}{\partial \hat{z}}\left(2 \hat{\mu} \frac{\partial \hat{w}}{\partial \hat{z}}\right)+\frac{1}{\hat{r}} \frac{\partial}{\partial \hat{r}}\left(\hat{\mu} \hat{r} \frac{\partial \hat{w}}{\partial \hat{r}}\right)+\frac{1}{\hat{r}} \frac{\partial}{\partial \hat{r}}\left(\hat{\mu} \hat{r} \frac{\partial \hat{u}}{\partial \hat{z}}\right)=0
\end{gathered}
$$

where $\hat{r}$ and $\hat{z}$ denote the radial and axial coordinates, $\hat{p}$ is the pressure within the molten glass, and $\hat{u}$ and $\hat{w}$ are the radial and axial velocities of the glass. The glass viscosity, denoted $\hat{\mu}$, is a function of its temperature $\hat{T}$ only, which may in general vary both along and across the fibre. At the centre of the fibre, symmetry provides the conditions

$$
\hat{u}=\frac{\partial \hat{w}}{\partial \hat{r}}=0,
$$

on $\hat{r}=0$, while the kinematic and dynamic boundary conditions are

$$
\begin{gathered}
\hat{u}=\hat{w} \frac{\mathrm{d} \hat{R}}{\mathrm{~d} \hat{z}}, \\
\left(-\hat{p}+2 \hat{\mu} \frac{\partial \hat{u}}{\partial \hat{r}}\right)=\hat{\mu} \frac{\mathrm{d} \hat{R}}{\mathrm{~d} \hat{z}}\left(\frac{\partial \hat{u}}{\partial \hat{z}}+\frac{\partial \hat{w}}{\partial \hat{r}}\right), \\
\hat{\mu}\left(\frac{\partial \hat{u}}{\partial \hat{z}}+\frac{\partial \hat{w}}{\partial \hat{r}}\right)=\frac{\mathrm{d} \hat{R}}{\mathrm{~d} \hat{z}}\left(-\hat{p}+2 \hat{\mu} \frac{\partial \hat{w}}{\partial \hat{z}}\right),
\end{gathered}
$$


Table I. Typical parameter values for the high-speed drawing of glass fibres, from ${ }^{1}$ Paek and Runk (1978), ${ }^{2}$ Myers (1989), ${ }^{3}$ Lee and Jaluria (1997), ${ }^{4}$ Yin and Jaluria (2000), ${ }^{5}$ Huang et al. (2008).

\begin{tabular}{lccc} 
Parameter & Symbol & Approximate value & Units \\
\hline & & & \\
tubing length $^{4,5}$ & $\hat{L}$ & 0.5 & $\mathrm{~m}$ \\
initial radius $^{4}$ & $\hat{R}_{0}$ & 0.05 & $\mathrm{~m}$ \\
input speed $^{4}$ & $\hat{W}_{0}$ & $10^{-3}$ & $\mathrm{~m} \mathrm{~s}^{-1}$ \\
draw speed $^{4}$ & $\hat{W}_{1}$ & 30 & $\mathrm{~m} \mathrm{~s}^{-1}$ \\
density $^{1,5}$ & $\hat{\rho}$ & 2200 & $\mathrm{~kg} \mathrm{~m}^{-3}$ \\
furnace temperature $^{2,3}$ & $\hat{T}_{f}$ & 3000 & $\mathrm{~K}$ \\
ambient air temperature $^{2,3}$ & $\hat{T}_{a}$ & 2250 & $\mathrm{~K}$ \\
glass softening temperature $^{2,3}$ & $\hat{T}_{s}$ & 1900 & $\mathrm{~K}^{-2}$ \\
softening viscosity $^{3}$ & $\hat{\mu}_{s}$ & 4500 & $\mathrm{~N} \mathrm{~s} \mathrm{~m}^{-2}$ \\
specific heat $^{1,3,5}$ & $\hat{c}_{p}$ & 1000 & $\mathrm{~J} \mathrm{~kg}^{-1} \mathrm{~K}^{-1}$ \\
heat transfer coefficient $^{1,3,5}$ & $\hat{k}_{h}$ & 100 & $\mathrm{~W} \mathrm{~m}^{-2}$ \\
thermal conductivity $^{3,5}$ & $\hat{k}_{c}$ & 1.1 & $\mathrm{~W} \mathrm{~m}^{-1} \mathrm{~K}^{-1}$ \\
Stefan-Boltzmann constant $^{3}$ & $\hat{\sigma}$ & $5.67 \times 10^{-8}$ & $\mathrm{~W} \mathrm{~m}^{-2} \mathrm{~K}^{-4}$ \\
specific emissivity $^{2}$ & $\varepsilon_{r}$ & 0.9 & - \\
refractive index $^{3}$ & $n_{0}$ & 1.5 & - \\
absorption coefficient $^{1,2}$ & $\hat{\chi}$ & 200 & $\mathrm{~m}^{-1}$ \\
\hline
\end{tabular}

on the glass-air interface $\hat{r}=\hat{R}(\hat{z})$, where we are neglecting any effects due to surface tension since these are typically small (Huang et al., 2008). The fluid problem is closed by specifying the velocity at the two ends of the tubing,

$$
\hat{w}(\hat{r}, \hat{z}=0)=\hat{W}_{0}, \quad \hat{w}(\hat{r}, \hat{z}=\hat{L})=\hat{W}_{1} .
$$

\subsubsection{Glass viscosity}

The viscosity of glass varies significantly with temperature, with a number of expressions for $\hat{\mu}(\hat{T})$ having been reported in the literature depending on the type of glass (Geyling and Homsy, 1980). We choose

$$
\hat{\mu}=\hat{\mu}_{s} \exp \left(a\left(\frac{1}{\hat{T}}-\frac{1}{\hat{T}_{s}}\right)\right)
$$

which is suitable for fused silica, where $\hat{T}_{s}$ is the softening temperature for glass, $\hat{\mu}_{s}$ the softening viscosity, and $a$ an empirically determined constant (Myers, 1989). 
2.1.3. Temperature equation

The equation governing the distribution of temperature is

$$
\hat{\rho} \hat{c}_{p}\left(\hat{u} \frac{\partial \hat{T}}{\partial \hat{r}}+\hat{w} \frac{\partial \hat{T}}{\partial \hat{z}}\right)=\frac{1}{\hat{r}} \frac{\partial}{\partial \hat{r}}\left(\hat{k}(\hat{T}) \hat{r} \frac{\partial \hat{T}}{\partial \hat{r}}\right)+\frac{\partial}{\partial \hat{z}}\left(\hat{k}(\hat{T}) \frac{\partial \hat{T}}{\partial \hat{z}}\right),
$$

where the left-hand side represents thermal convection and the righthand side thermal conduction, with $\hat{k}(\hat{T})$ denoting the conductivity of glass. For transparent materials, radiative transfer within the material is of significance, especially at high temperatures. A full description of this effect is a formidable challenge; however provided the fibre is optically thick, that is, $\hat{R}$ is much greater than the absorption lengthscale $1 / \hat{\chi}$, where $\hat{\chi}$ is the glass absorption coefficient (with typical values given in Table I) one may use the Rosseland approximation to include a radiative contribution to the thermal conductivity, $\hat{k}_{c}$, so that the apparent conductivity $\hat{k}(\hat{T})=\hat{k}_{c}+\hat{k}_{r}(\hat{T})$, where (Paek and Runk, 1978)

$$
\hat{k}_{r}(\hat{T})=\frac{16 n_{0}^{2} \hat{\sigma} \hat{T}^{3}}{3 \hat{\chi}} .
$$

Here $\hat{\sigma}$ is the Stefan-Boltzmann constant and $n_{0}$ denotes the refractive index for the glass (with typical values given in Table I). We may therefore separate the respective conductive components in (4) to give

$$
\begin{aligned}
\hat{\rho} \hat{c}_{p}\left(\hat{u} \frac{\partial \hat{T}}{\partial \hat{r}}+\hat{w} \frac{\partial \hat{T}}{\partial \hat{z}}\right) & =\frac{1}{\hat{r}} \frac{\partial}{\partial \hat{r}}\left(\hat{k}_{c} \hat{r} \frac{\partial \hat{T}}{\partial \hat{r}}\right)+\frac{\partial}{\partial \hat{z}}\left(\hat{k}_{c} \frac{\partial \hat{T}}{\partial \hat{z}}\right) \\
& +\frac{1}{\hat{r}} \frac{\partial}{\partial \hat{r}}\left(\hat{\kappa}_{r}(\hat{T}) \hat{r} \frac{\partial \hat{T}^{4}}{\partial \hat{r}}\right)+\frac{\partial}{\partial \hat{z}}\left(\hat{\kappa}_{r}(\hat{T}) \frac{\partial \hat{T}^{4}}{\partial \hat{z}}\right)
\end{aligned}
$$

where $\hat{\kappa}_{r}(\hat{T})=\hat{k}_{r} / 4 \hat{T}^{3}$. The assumption may break down once the fibre is drawn down to a thickness of $O(100 \mu \mathrm{m})$, at which point one might expect the glass to absorb the radiation directly from the surrounding furnace and re-radiate heat back to the furnace. $\dagger$ Nevertheless, given that the absorption lengthscale is highly dependent on the glass properties and radiation wavelength, in the interest of simplicity we limit ourselves to considering only the optically thick limit. Furthermore, we note that the validity of the Rosseland approximation for the fibre drawing process has been confirmed by numerical tests using more realistic models for bulk radiation (Yin and Jaluria, 1997).

$\dagger$ We note that one can tackle this optically thin limit in an ad hoc manner by including an additional radiative term in the temperature equation (4), as done by Fitt et al. (2002). 
The boundary condition at the glass-air interface $\hat{r}=\hat{R}(\hat{z})$ is

$$
-\hat{k} \frac{\partial \hat{T}}{\partial \hat{r}}=\hat{\sigma} \varepsilon_{r}\left(\hat{T}^{4}-\hat{T}_{f}^{4}\right)+\hat{k}_{h}\left(\hat{T}-\hat{T}_{a}\right),
$$

where $\hat{T}_{f}=\hat{T}_{f}(\hat{z})$ and $\hat{T}_{a}=\hat{T}_{a}(\hat{z})$ are, respectively, the temperatures of the furnace and ambient air in the furnace, both of which are assumed to be known functions of the distance along the furnace, as discussed in $\S 2.1 .4$. The terms on the right-hand side represent respectively radiative transfer and convective heating to the surroundings via Newton cooling (Carslaw and Jaeger, 1959). Here $\varepsilon_{r}$ is the specific emissivity and $\hat{k}_{h}$ is the heat transfer coefficient, which may depend on the radius of the fibre; appropriate functional forms are discussed in $\S 2.1 .5$.

Finally, the system is closed by specifying the input temperature $\hat{T}=\hat{T}_{0}$ at $\hat{z}=0$. For simplicity, we assume that $\hat{T}_{0}$ is a constant so that the heat flux through the outer radius is zero. Then (7) implies that $\hat{T}_{0}$ is given by the solution to the quartic equation

$$
\hat{\sigma} \varepsilon_{r}\left(\hat{T}_{0}^{4}-\hat{T}_{f}(0)^{4}\right)+\hat{k}_{h}\left(\hat{T}_{0}-\hat{T}_{a}(0)\right)=0 .
$$

However we note that, in reality, the temperature of the glass entering the furnace is unlikely to satisfy (8), rather it will come in at the far lower ambient temperature outside the furnace, say around $300 \mathrm{~K}$. In this case we expect there to be a small transient near the inlet over which the system quickly adjusts to the solution of (8). We investigate this thermal boundary layer in $\S$ 4.1.1.

2.1.4. The form of $\hat{T}_{f}$ and $\hat{T}_{a}$

The furnace temperature $\hat{T}_{f}$ is not very well characterized, but it is known that the furnace is generally hottest in the central portion and coolest at the ends (Lee and Jaluria, 1997), and has been typically modelled as having either a Gaussian or parabolic profile (Roy Choudhury and Jaluria, 1998). In order to evaluate the effect of the furnace temperature, we consider both an isothermal and varying temperature profile, with the latter taken to be of the functional form (Filippov, 2011)

$$
\hat{T}_{f}(\hat{z})=\hat{T}_{m}\left(\frac{1}{5}+\frac{4}{5} \mathrm{e}^{-0.5(\hat{z} / \hat{L}-0.35)^{2}}\right),
$$

with the maximum temperature $\hat{T}_{m} \approx 3000 \mathrm{~K}$.

For a given furnace profile, the ambient air temperature should be determined as part of the solution to the full heat transfer problem (Roy Choudhury et al., 1999). However, for simplicity we assume the 
ambient temperature to be known. Several authors take this to be constant, with $\hat{T}_{a} \approx \hat{T}_{m} / 2$ (Paek and Runk, 1978, Lee and Jaluria, 1997) or equal to room temperature (Huang et al., 2008). However, full numerical simulations suggest that $\hat{T}_{a}(\hat{z})$ follows the same profile as $\hat{T}_{f}(\hat{z})$, so we follow Filippov (2011) and set $\hat{T}_{a}(\hat{z})=3 \hat{T}_{f}(\hat{z}) / 4$ throughout.

2.1.5. The form of $\varepsilon_{r}$ and $\hat{k}_{h}$

Although typical values for the specific emissivity $\varepsilon_{r}$ and heat transfer coefficient $\hat{k}_{h}$ are given in Table I and these parameters are often treated as constant, they are in fact both dependent on the material properties, temperature, and radius of the fibre. The emissivity is particularly tricky to measure at typical furnace temperatures, and is usually assumed to behave as if at room temperature, where it is experimentally found to decrease with decreasing fibre radius. While Paek and Runk (1978) posed an ad hoc smoothly varying $\varepsilon_{r}(\hat{R}) \in[0.1,0.6]$, Myers (1989) derived a more accurate model with $\varepsilon_{r}(\hat{R}) \in(0,0.9]$, reaching the higher value for $\hat{R} \gtrsim 1 / \hat{\chi} \approx 10^{-2} \mathrm{~m}$.

To maintain generality we consider separately the cases of an emissivity that is constant and one that is of the functional form $\varepsilon_{r} \sim 1-\mathrm{e}^{-\phi \hat{R}}$, with $\phi \approx 2.5 \hat{\chi}$ chosen so as to give qualitative agreement with Myers's model.

The heat transfer coefficient $\hat{k}_{h}$ is generally thought to vary only weakly throughout the process, although values between $10-300 \mathrm{Wm}^{-2}$ have been reported in the literature. While it is generally taken to be constant, Geyling and Homsy (1980) and Forest and Zhou (2001) have assumed the functional form $\hat{R} \hat{k}_{h} \sim(\hat{w} \hat{R})^{1 / 3}$, derived by considering flow past a cylinder. In this paper we therefore investigate the effect of a $\hat{k}_{h}$ that is both constant and of the form posed by Geyling and Homsy.

\subsection{NON-DIMENSIONALIZATION}

We exploit the slenderness of the geometry, that is, we introduce the inverse aspect ratio $\epsilon=\hat{R}_{0} / \hat{L} \approx 0.1 \ll 1$, and scale using

$$
\begin{array}{llll}
\hat{r}=\epsilon \hat{L} r, & \hat{z}=\hat{L} z, & \hat{u}=\epsilon \hat{W}_{0} u, & \hat{w}=\hat{W}_{0} w, \\
\hat{R}=\epsilon \hat{L} R, & \hat{T}=\hat{T}_{s} T, & \hat{p}=\frac{\hat{\mu}_{s} \hat{W}_{0}}{\epsilon^{2} \hat{L}} p, & \hat{\mu}=\hat{\mu}_{s} \mu .
\end{array}
$$


Substituting into (1) and (4) provides the dimensionless system

$$
\begin{gathered}
\frac{1}{r} \frac{\partial}{\partial r}(r u)+\frac{\partial w}{\partial z}=0 \\
-\frac{\partial p}{\partial r}+\epsilon^{4} \frac{\partial}{\partial z}\left(\mu \frac{\partial u}{\partial z}\right)+\epsilon^{2} \mu \frac{\partial}{\partial r}\left(\frac{1}{r} \frac{\partial}{\partial r}(r u)\right) \\
+\epsilon^{2} \frac{\partial \mu}{\partial z} \frac{\partial w}{\partial r}+2 \epsilon^{2} \frac{\partial \mu}{\partial r} \frac{\partial u}{\partial r}=0 \\
-\frac{\partial p}{\partial z}+\epsilon^{2} \frac{\partial}{\partial z}\left(2 \mu \frac{\partial w}{\partial z}\right)+\frac{1}{r} \frac{\partial}{\partial r}\left(\mu r \frac{\partial w}{\partial r}\right)+\frac{\epsilon^{2}}{r} \frac{\partial}{\partial r}\left(\mu r \frac{\partial u}{\partial z}\right)=0, \\
\epsilon^{2} P e\left(u \frac{\partial T}{\partial r}+w \frac{\partial T}{\partial z}\right)=\frac{1}{r} \frac{\partial}{\partial r}\left(r \frac{\partial T}{\partial r}+\gamma r \frac{\partial T^{4}}{\partial r}\right)+\epsilon^{2} \frac{\partial^{2}}{\partial z^{2}}\left(T+\gamma \frac{\partial T^{4}}{\partial z}\right),
\end{gathered}
$$

where $P e=\hat{\rho} \hat{c}_{p} \hat{W}_{0} \hat{L} / \hat{k}_{c}$ is the Péclet number and $\gamma=4 n_{0}^{2} \hat{\sigma} \hat{T}_{s}^{3} / 3 \hat{\chi} \hat{k}_{c}$ measures the importance of bulk diffusion. The dimensionless boundary conditions are

$$
u=\frac{\partial w}{\partial r}=0
$$

on $r=0$, and

$$
\begin{aligned}
u & =w \frac{\mathrm{d} R}{\mathrm{~d} z}, \\
-p+2 \epsilon^{2} \mu \frac{\partial u}{\partial r} & =\epsilon^{2} \mu \frac{\mathrm{d} R}{\mathrm{~d} z}\left(\epsilon^{2} \frac{\partial u}{\partial z}+\frac{\partial w}{\partial r}\right), \\
\mu\left(\epsilon^{2} \frac{\partial u}{\partial z}+\frac{\partial w}{\partial r}\right) & =\frac{\mathrm{d} R}{\mathrm{~d} z}\left(-p+2 \epsilon^{2} \mu \frac{\partial w}{\partial z}\right), \\
-\left(1+4 \gamma T^{3}\right) \frac{\partial T}{\partial r} & =\epsilon \alpha\left(T^{4}-T_{f}^{4}\right)+\epsilon \beta\left(T-T_{a}\right),
\end{aligned}
$$

on $r=R(z)$, where $\alpha=\hat{\sigma} \varepsilon_{r} \hat{T}_{s}^{3} \hat{L} / \hat{k}_{c}$ and $\beta=\hat{k}_{h} \hat{L} / \hat{k}_{c}$ represent the importance of conduction relative to radiation and convection respectively. We note that, when only one of these effects is accounted for, both $\alpha$ and $\beta$ have been referred to as the Biot number (Wylie et al., 2007, Huang et al., 2008). Typical values are given in Table II, although we note that these may vary both with the fibre radius and the type of glass used. Finally we have boundary conditions on the fibre temperature and velocity at input and the pulling speed as the fibre exits the furnace, namely

$$
w(r, 0)=1, \quad T(r, 0)=T_{0}, \quad w(r, 1)=w_{1},
$$


Table II. Dimensionless parameter definitions and approximate values.

\begin{tabular}{lcc} 
Parameter & Symbol & Approximate value \\
\hline Aspect ratio & $\epsilon=\hat{R}_{0} / \hat{L}$ & 0.1 \\
Reynolds number & $R e=\frac{\hat{\rho} \hat{W}_{0} \hat{L}}{\hat{\mu}_{s}}$ & $\lesssim 10^{-3}$ \\
Péclet number & $P e=\frac{\hat{\rho} \hat{c}_{p} \hat{W}_{0} \hat{L}}{\hat{k}_{c}}$ & 1000 \\
& $w_{1}=\frac{\hat{W}_{1}}{\hat{W}_{0}}$ & $3 \times 10^{4}$ \\
Draw ratio & $\alpha=\frac{\hat{\sigma} \varepsilon_{r} \hat{T}_{s}^{3} \hat{L}}{\hat{k}_{c}}$ & 160 \\
Surface radiation parameter & $\beta=\frac{\hat{k}_{h} \hat{L}}{\hat{k}_{c}}$ & \\
Conduction parameter & $\gamma=\frac{4 n_{0}^{2} \hat{\sigma} \hat{T}_{s}^{3}}{3 \hat{\chi} \hat{k}_{c}}$ & 50 \\
Bulk radiation parameter & &
\end{tabular}

where the draw ratio $w_{1}=\hat{W}_{1} / \hat{W}_{0}$ is the ratio of axial draw speed to input speed and $T_{0}=\hat{T}_{0} / T_{s}$ is the dimensionless inlet temperature. Finally, from (3) and taking $a \approx 61000 \mathrm{~K}$ (Myers, 1989) we find the dimensionless viscosity

$$
\mu(T)=\exp (32(1 / T-1))
$$

\section{Fluid flow}

Seeking regular parameter expansions of the form $u=u^{(0)}+\epsilon^{2} u^{(1)}+\cdots$ into the flow problem, $(11 a-c)$ and $(12 a-c)$, and considering the resulting equations to leading order, we find that $w^{(0)}=w^{(0)}(z)$ and thus the flow is extensional. A similar approach to Cummings and Howell (1999) 
may then be used to derive the following leading-order equations

$$
\begin{aligned}
\frac{\mathrm{d}}{\mathrm{d} z}\left(w^{(0)} R^{(0)^{2}}\right) & =0, \\
\frac{\mathrm{d}}{\mathrm{d} z}\left(3 \bar{\mu}^{(0)} R^{(0)^{2}} \frac{\mathrm{d} w^{(0)}}{\mathrm{d} z}\right) & =0,
\end{aligned}
$$

representing conservation of mass and an axial stress balance respectively, where overbars denote the radially averaged quantity

$$
\bar{\phi}(z)=\frac{2}{R^{2}} \int_{0}^{R} r \phi(r, z) \mathrm{d} r .
$$

Integrating (15a-b) and applying boundary condition (13f) yields

$$
\begin{aligned}
w^{(0)} R^{(0)^{2}} & =1, \\
3 \bar{\mu}^{(0)} R^{(0)^{2}} \frac{\mathrm{d} w^{(0)}}{\mathrm{d} z} & =F,
\end{aligned}
$$

where $F$ is the (constant) tension in the fibre. In our problem we prescribe the draw speed $w(1)=w_{1}$ (chosen here to be $3 \times 10^{4}$ which corresponds to a high draw speed of $\left.30 \mathrm{~ms}^{-1}\right)$. Then $F$ is determined as part of the solution once we have found the temperature and hence radially-averaged viscosity $\bar{\mu}^{(0)}$ using (14) and (16). We discuss the variation of $F$ with $w_{1}, \alpha$ and $\beta$ in $\S 4.1 .3$.

\section{Temperature profiles}

The temperature system $(11 d)$ and $(12 e)$ possesses various asymptotic limits of interest and we address these below. We note that several of the dimensional parameters given in Table I, and thus their dimensionless counterparts given in Table II, are uncertain and may vary by up to an order of magnitude depending on the glass used, and so the different limits will be applicable to these different scenarios.

\subsection{RAPID HeAt transport ACROSS THE FIBRE RADIUS}

\subsubsection{Surface radiation and conduction balance axial convection}

We begin by supposing that $P e=P e^{*} / \epsilon$ where $P e^{*}=O(1)$, and also assume $\alpha, \beta$, and $\gamma$ to be of order unity. In practice this corresponds to high conduction, so that it balances both with surface radiation and axial convection. Substituting a regular parameter expansion of the form $T=T^{(0)}+\epsilon T^{(1)}+\cdots$ into $(11 d)$ and using (12e) indicates that 
$T^{(0)}=T^{(0)}(z)$, and thus there are no variations in temperature across the fibre. The system is closed by proceeding to second order in (11d), integrating over the fibre radius and using (12e) and (17a), leading to the system

$$
\begin{aligned}
3 \bar{\mu}^{(0)}\left(T^{(0)}\right) \frac{\mathrm{d} w^{(0)}}{\mathrm{d} z} & =F w^{(0)} \\
\frac{1}{2} \sqrt{w^{(0)}} P e^{*} \frac{\mathrm{d} T^{(0)}}{\mathrm{d} z} & =-\left(\alpha\left(T^{(0)^{4}}-T_{f}^{4}\right)+\beta\left(T^{(0)}-T_{a}\right)\right),
\end{aligned}
$$

subject to boundary conditions

$$
w^{(0)}(r, 0)=1, \quad T^{(0)}(r, 0)=T_{0}, \quad w^{(0)}(r, 1)=w_{1} .
$$

The evolution of temperature, viscosity, axial velocity, and radius with axial position, taking the illustrative parameter choice $P e^{*}=1$, for different (but constant in space) values of $\alpha$ and $\beta$ are shown in Fig. 2, where we have assumed the Gaussian furnace temperature profile (9). We see that the viscosity varies through many orders of magnitude as the fibre is drawn through the furnace. The fibre temperature is heavily guided by the furnace temperature, peaking around or just after the furnace peak temperature. Following this, the temperature then falls throughout the remainder of the furnace. The axial velocity and fibre radius both vary over a relatively small axial distance, corresponding to the region where the glass temperature is near its maximum.

We notice that the axial velocity of the glass dramatically increases as we move down the profile. As a result it is useful to visualize the temperature profile of a material element of molten glass as it moves through the furnace. This corresponds to determining the temperature as a function of a dimensionless 'time variable', $\tau=\tau(z)$, defined by

$$
\tau=\int_{0}^{z} \frac{1}{w^{(0)}(\zeta)} \mathrm{d} \zeta
$$

which we identify with the time at which an element starting at the top of the furnace reaches the axial position $z$. The rapid acceleration of the glass as it moves towards the end of the furnace is clearly shown by the relation between $\tau$ and axial position $z$ in Fig. 3(a). The rapid cooling is evident in Figs. 3(b)-(d), where we show the temperature, viscosity, and fibre radius varying dramatically in the final stages of the drawing process.

Aside from exhibiting all of the general features that are found in practice, these examples also illustrate the effect of $\alpha$ and $\beta$ on the process: a higher $\beta$ leads to stronger cooling, while a higher $\alpha$ is needed to raise the glass temperature to the furnace temperature. In 


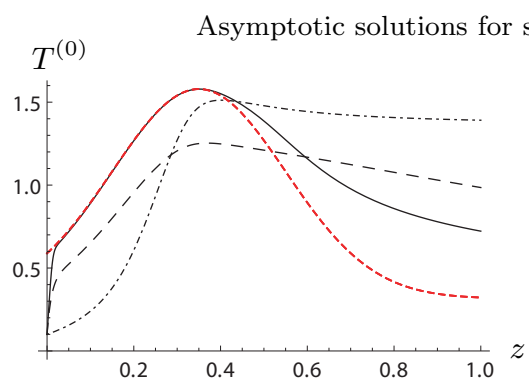

(a)

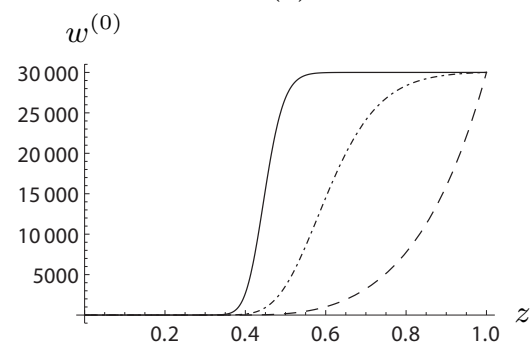

(c)

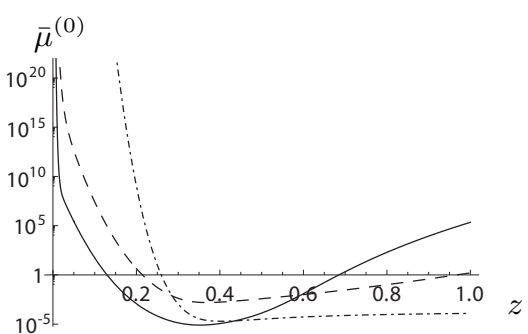

(b)

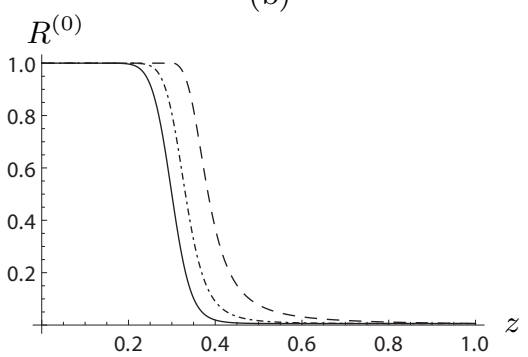

(d)

Figure 2. Effect of varying furnace temperature on the leading-order variation of (a) temperature, (b) viscosity, (c) axial velocity, and (d) radius, with axial position for $P e^{*}=1, w_{1}=3 \times 10^{4}, T_{a}=3 T_{f} / 4, T_{0}=T_{s} / 10$. In all cases the black solid line illustrates the case when $\alpha=160, \beta=1$, the dot-dashed line shows the case $\alpha=1, \beta=50$, and the dashed line shows the case $\alpha=1, \beta=1$. The red dashed line in (a) shows the furnace temperature $T_{f}(z)$, given by (9) with $T_{m}=3000 \mathrm{~K}$.

order to examine this more closely, we consider the case of a constant furnace temperature $T_{f}$ in Fig. $4 . \ddagger$ These plots clearly illustrate the initial boundary layer in which the glass temperature rapidly increases from $T_{0}$ to its equilibrium value $T_{e}$ given by the solution to

$$
\alpha\left(T_{e}^{4}-T_{f}^{4}\right)+\beta\left(T_{e}-T_{a}\right)=0 .
$$

Furthermore, we see that the boundary layer width decreases with increasing $\alpha$, while changing $\beta$ affects only the steady state value attained. For the large values of $\alpha$ typically observed in practice, we are therefore justified in ignoring this initial transient and setting $T_{0}=T_{e}$ as the initial condition for the temperature.

\footnotetext{
$¥$ We note that the constant $T_{f}$ case is amenable to phase-plane analysis, of which details may be found in the Appendix.
} 


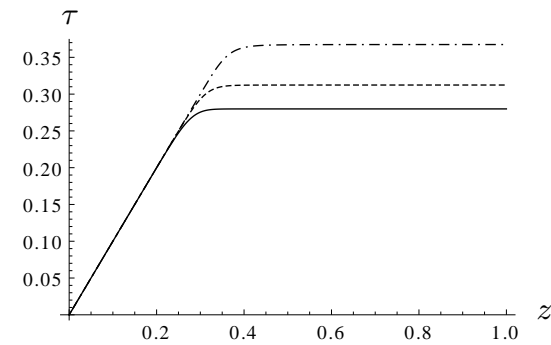

(a)

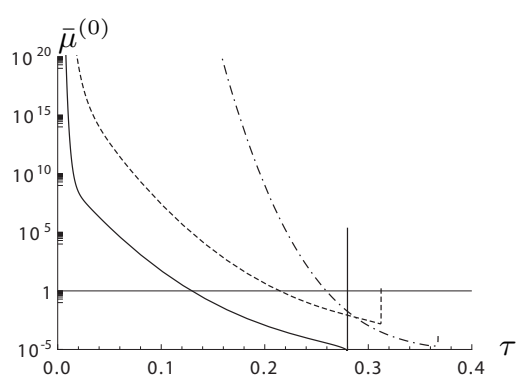

(c)

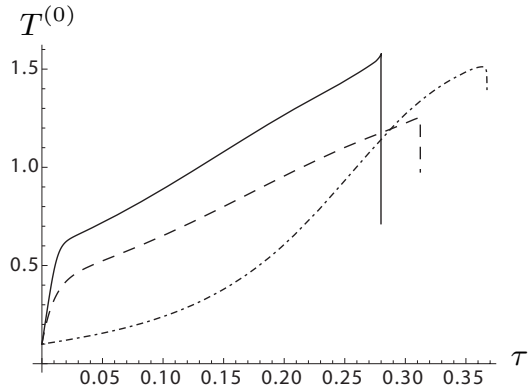

(b)

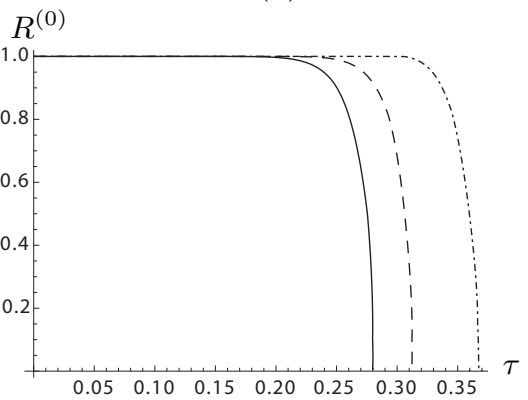

(d)

Figure 3. (a) Time variable $\tau$ against axial distance $z$ for $\alpha=160, \beta=1$ (solid line), $\alpha=1, \beta=50$, (dot-dashed line) and $\alpha=1, \beta=1$ (dashed line). The corresponding (b) temperature, (c) viscosity, and (d) radius are plotted against $\tau$. In all cases $P e^{*}=1, w_{1}=3 \times 10^{4}, T_{a}=3 T_{f} / 4, T_{0}=T_{s} / 10$.

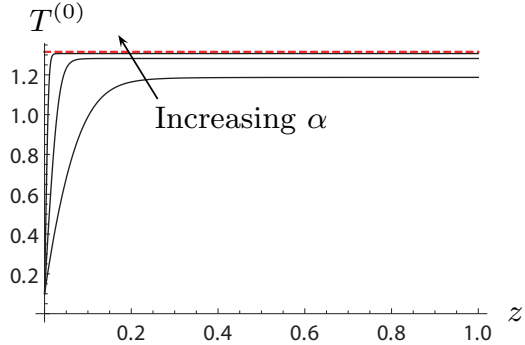

(a)

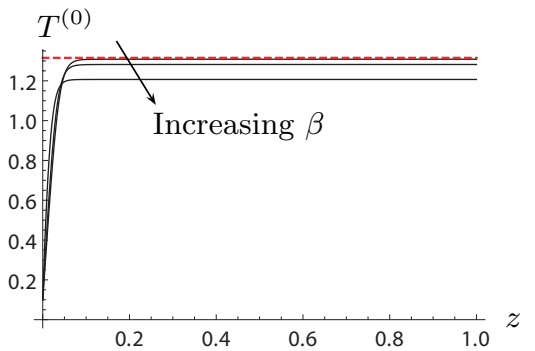

(b)

Figure 4. Influence of (a) $\alpha$ and (b) $\beta$ on the variation of glass temperature with axial position taking a constant furnace temperature $T_{f}=2500 \mathrm{~K}$, shown red (dashed). In (a) we take $\beta=5, \alpha=\{1,5,20\}$, while in (b) $\alpha=1, \beta=\{1,5,20\}$. In all cases $P e^{*}=1, w_{1}=3 \times 10^{4}, T_{a}=3 T_{f} / 4, T_{0}=T_{s} / 10$. 
4.1.2. The effect of $\alpha(z), \beta(z)$

We now consider the effect of letting $\alpha$ and $\beta$ vary in the axial direction. Following our discussion in $\S 2.1 .5$, we take $\varepsilon_{r} \sim 1-\mathrm{e}^{-\phi \hat{R}}$ with $\phi=2.5 \hat{\chi}$ and use $(17 a)$ to obtain

$$
\alpha(z)=\alpha_{0}\left(1-\mathrm{e}^{-25 / \sqrt{w^{(0)}(z)}}\right),
$$

where $\alpha_{0}$ is a constant. Taking $\hat{R} \hat{k}_{h} \sim(\hat{w} \hat{R})^{1 / 3}$ as discussed in $\S 2.1 .5$ and using (17a) leads to

$$
\beta(z)=\beta_{0} w^{(0)^{2 / 3}}
$$

where $\beta_{0}$ is a constant.

We compare the difference between constant $\alpha$ and $\alpha(z)$ (with $\beta$ held constant) in Fig. 5(a), where we see that a lower glass temperature is taken on exiting the furnace when $\alpha$ is a constant than when alpha varies with axial position. We note that, when $\alpha$ depends upon axial position, Newton cooling is the dominant mechanism in the later stages of the process, as argued by, for example, Huang et al. (2008). Data for the exit glass fibre temperature would provide a mechanism to determine the most suitable form for $\alpha$.

On the other hand, we see in Fig. 5(b) that, with the proposed functional form for $\beta(z)$, the temperature drops off before the furnace reaches its maximum temperature and the glass viscosity is not reduced enough to enable effective drawing. We therefore believe that this form is not realistic, and that $\beta$ should be kept constant. This is in agreement with the numerical results to the full conjugate heat transfer problem which reported that $\hat{k}_{h}$ remained approximately constant throughout the process (Yin and Jaluria, 2000).

For the remainder of this paper we focus our attention on the case when $\alpha$ and $\beta$ are constant.

\subsubsection{The force}

An important quantity for industrial applications is the force, $F$, required to draw the fibre, found as part of solution to (18), satisfying $F=3 \log w_{1} / \int_{0}^{1} 1 / \bar{\mu}^{(0)} \mathrm{d} z$. We plot the force as a function of the prescribed draw ratio $w_{1}$ in Fig. 6(a), which as expected is a monotonically increasing function. We also plot the force against $\alpha$, and $\beta$ in Figs. 6(b)-(c); interestingly the former is found to be non-monotonic at low values of $\alpha$.

We note that, for a rapidly varying choice of $\mu(T)$, Wylie et al. (2007) reported that a simplified model with constant heating and no cooling could predict three possible branches in force/draw-ratio space, 


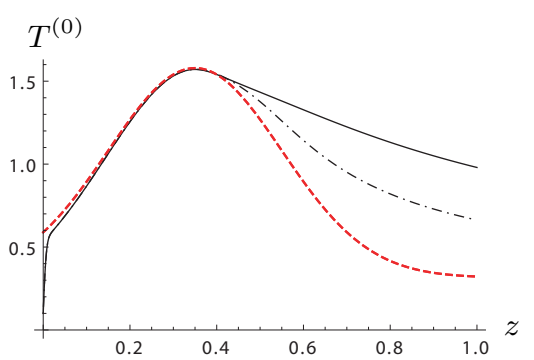

(a)

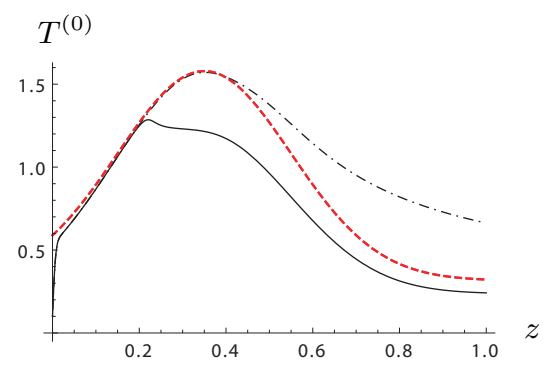

(b)

Figure 5. Influence of (a) $\alpha(z)$ given by Eqn. (22a), solid curve, and (b) $\beta(z)$ given by Eqn. $(22 b)$, solid curve, compared to constant values $\alpha_{0}=160$ and $\beta_{0}=50$, shown dot-dashed; the furnace temperature is also shown (dashed). In all cases $P e^{*}=1$, $w_{1}=3 \times 10^{4}, T_{a}=3 T_{f} / 4, T_{0}=T_{s} / 10$.

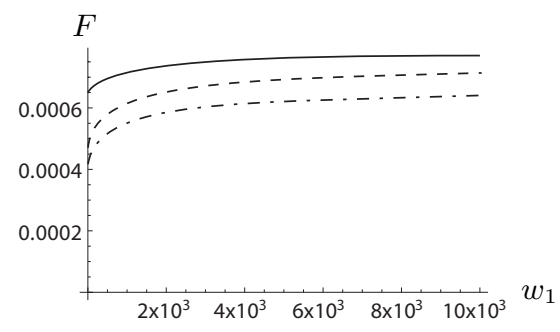

(a)

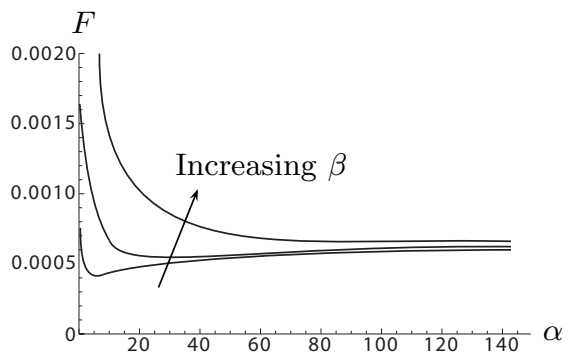

(b)

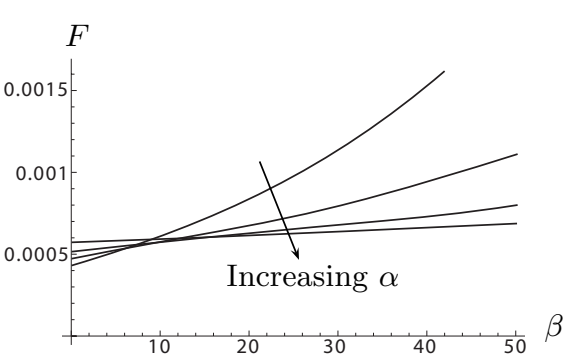

(c)

Figure 6. Force $F$ against (a) $w_{1}$ for $\alpha=160, \beta=50$ (solid), $\alpha=1, \beta=50$ (dashed), $\alpha=1, \beta=1$ (dot-dashed); (b) $\alpha$ for $\beta=\{1,10,50\}, w_{1}=3 \times 10^{4}$; (c) $\beta$ for $\alpha=\{10,20,50,100\}, w_{1}=3 \times 10^{4}$. In all cases $P e^{*}=1, T_{a}=3 T_{f} / 4, T_{0}=T_{s} / 10$.

two of which were potentially stable. This surprising result was later extended to include surface tension and inertial effects (Suman and Kumar, 2009). Although we have also been able to reproduce their results for our model using the $\mu(T)$ form suggested by Wylie et al. (2007), we note that the solutions are very sensitive to the parameter values; in particular we have not found the three branches for our choice of $\mu(T)$ and range of realistic parameter values. 


\subsubsection{Surface radiation and conduction dominate}

Our parameter estimates given in Table I suggest that both $\alpha$ and $\beta$ may in fact be quite large. If we therefore assume that $\alpha=\lambda \alpha^{*}$ and $\beta=\lambda \beta^{*}$ where $\alpha^{*}, \beta^{*}$ are of order unity and $\lambda \gg 1$, then surface radiation conduction dominate over axial convection and we may neglect the left-hand side of $(18 b)$. If we now assume that $\alpha^{*}$ and $\beta^{*}$ are constant, then $T^{(0)}(z)$ is given simply by $\mathcal{T}(z)$, the solution to the quartic equation

$$
\alpha^{*}\left(\mathcal{T}(z)^{4}-T_{f}(z)^{4}\right)+\beta^{*}\left(\mathcal{T}(z)-T_{a}(z)\right)=0,
$$

which may be expressed explicitly although, due to its complicated form, we refrain from writing it here. Once determined, (18a) may be integrated to give

$$
w^{(0)}=\exp \left(\int_{0}^{z} \frac{3 F}{\bar{\mu}^{(0)}(T(s))} \mathrm{d} s\right)
$$

with $F$ chosen so that $w^{(0)}(1)=w_{1}$, and $R^{(0)}$ may then be calculated from $(17 a)$.

Towards the end of the furnace the fibre velocity rises significantly and the term neglected on the left-hand side of (18b) becomes important again; a boundary layer is thus present. Rescaling $w^{(0)}=\lambda^{2} \mathcal{W}$ we find that the system $(18 b)$ now reads

$$
\frac{1}{2} \sqrt{\mathcal{W}} P e^{*} \frac{\mathrm{d} T^{(0)}}{\mathrm{d} z}=-\left(\alpha^{*}\left(T^{(0)^{4}}-T_{f}^{4}\right)+\beta^{*}\left(T^{(0)}-T_{a}\right)\right),
$$

and we return to solving the original full system (18). However, since the numerical simulations indicate that the velocity evolves to its final value over a fairly narrow window (near where the temperature attains its maximum) its value is approximately constant (and equal to the draw speed) for an appreciable portion of the fibre drawing. We thus propose that we may set $\mathcal{W}_{1}=W_{1} / \lambda^{2}(=O(1))$, the (known) fibre pulling speed, so that (25) provides a decoupled autonomous equation for $T^{(0)}$ in this region which may be solved implicitly to give

$$
z\left(T^{(0)}\right)=\int_{1}^{T^{(0)}}-\frac{\sqrt{W_{1}} P e^{*} \mathrm{~d} s}{\left(\alpha^{*}\left(s^{4}-T_{f}^{4}\right)+\beta^{*}\left(s-T_{a}\right)\right)} .
$$

We may then patch the solution to (23) with the solution to (26) to find an approximation to the full temperature distribution. Once determined, $w^{(0)}$ and $R^{(0)}$ are easily calculated from (18a) and (17a) as before. This patched asymptotic solution is compared with the solution 




(a)

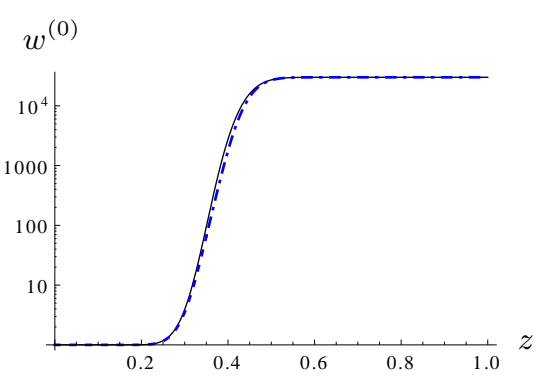

(b)

Figure 7. (a) Variation of leading-order temperature with axial position given by the full system (18) when $P e=1, \alpha=160$ and $\beta=50$ (black), while the blue dashed line shows the asymptotic solution $\mathcal{T}$ given by (23). In both cases $P e^{*}=1, w_{1}=3 \times 10^{4}$, $T_{a}=3 T_{f} / 4, T_{0}=T_{e}$. In (b) the corresponding numerical and asymptotic solutions for the fibre radius are shown.

to the full coupled system (18) in Fig. 7, showing excellent agreement, with the two solutions almost indistinguishable. We note that, to complete a formal asymptotic analysis results in the need to solve the full problem in a small region that matches between the solution determined in the two regions here. Nevertheless, the asymptotic solutions derived here provide a simple yet accurate description of the system behaviour.

\subsubsection{First-order correction}

The $\alpha, \beta$ limit discussed in $\S 4.1 .4$ may be treated in an asymptotically rigorous manner by setting $\alpha=\alpha^{*} / \epsilon^{p}, \beta=\beta^{*} / \epsilon^{q}$, where $p, q \in \mathbb{Z}_{+}$. Since the leading-order system depends only on axial position, to quantify the radial variations we must analyse the system at the next order in the expansion in powers of $\epsilon$. In doing so, provided $w^{(0)}$ is of order unity, which we have seen is true up to the point at which the glass reaches its maximum temperature, we may use Eqn. (11d) and boundary condition $(12 e)$ at $O(\epsilon)$ (recalling that $\left.\epsilon P e=P e^{*}\right)$ to give

$$
T(r, z)=\mathcal{T}(z)+\epsilon\left[\frac{P e^{*}\left(r^{2}-R^{(0)^{2}}\right) w^{(0)} \mathcal{T}^{\prime}(z)}{4\left(1+4 \gamma \mathcal{T}(z)^{3}\right)}+A(z)\right]
$$




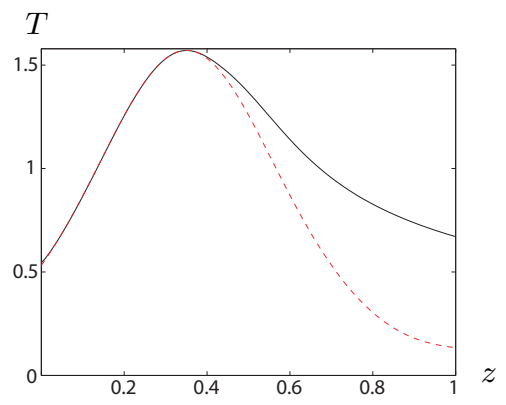

(a)

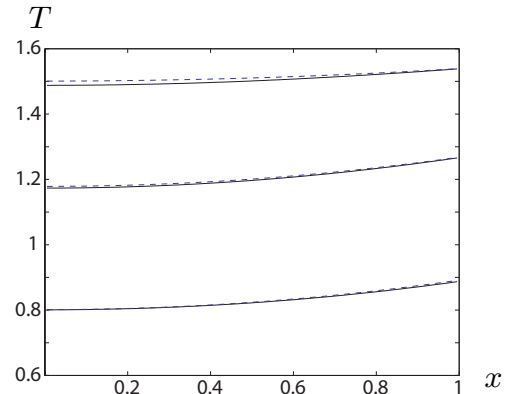

(b)

Figure 8. Numerical (solid) and asymptotic (dashed) results for the temperature against (a) axial position, with $x=0$, and (b) scaled radial position, $x$, with $z=\{0.1,0.2,0.3\}$, taking $\epsilon=0.1, P e^{*}=1, \alpha=1.6 / \epsilon^{2}, \beta=5 / \epsilon, \gamma=0, w_{1}=3 \times 10^{4}$, $T_{a}=3 T_{f} / 4$, and $T_{0}=T_{e}$.

Here, $A(z)$ depends on the size of $p$ and $q$ : provided $\alpha^{*}$ and $\beta^{*}$ are kept constant, we find from $(12 e)$ that

$$
A(z)=\left\{\begin{array}{cl}
-\frac{P e^{*} w^{(0)} \mathcal{T}^{\prime}(z) R}{2\left(\beta^{*}+4 \alpha^{*} \mathcal{T}(z)^{3}\right)}, & p=q=1, \\
-\frac{\beta^{*}\left(T_{f}-T_{a}\right)}{4 T_{f}^{3}}, & p=2, q=1, \\
-\frac{\alpha^{*}}{\beta^{*}} \frac{1}{\left(T_{a}^{4}-T_{f}^{4}\right)}, & p=1, q=2, \\
0, & p, q \geq 2 .
\end{array}\right.
$$

We plot the temperature profile (27) against both axial position and scaled radial position, $x(r, z)=r / R^{(0)}(z)$, for the case $p=2$, $q=1$ in Fig. 8, showing excellent agreement with the numerical solution to the full two-dimensional problem (see $\S 4.2$ ), at least until the temperature reaches its maximum. After this point the axial velocity $w^{(0)}$ increases rapidly and we can no longer ignore the left-hand side of (11d). Nevertheless, these asymptotic results give us a useful check on our two-dimensional numerical solution, which we now discuss.

\subsection{Transport across the Fibre Balancing CONVECTION}

\subsubsection{Surface radiation and conduction balance convection}

We now let $P e=O\left(1 / \epsilon^{2}\right)=\mathcal{P} / \epsilon^{2}$, which we note is likely to be relevant to fibre drawing at the higher speeds that have become possible more recently (Yin and Jaluria, 2000). In this case, heat transfer across the fibre is balanced by the convective transport and, from (11d) we see 
that $T^{(0)}$ depends on both $r$ and $z$. However, it is convenient to change variables into a coordinate frame that adapts to the radius of the fibre, via

$$
x(r, z)=\frac{r}{R^{(0)}(z)}, \quad \quad \zeta=\mathcal{P} z .
$$

This transforms the system (11d) and (12e) into

$$
\frac{\partial T^{(0)}}{\partial \zeta}=\frac{1}{x} \frac{\partial}{\partial x}\left(x \frac{\partial T^{(0)}}{\partial x}\left(1+4 \gamma T^{(0)^{3}}\right)\right)
$$

with

$$
-\frac{\partial T^{(0)}}{\partial x}\left(1+4 \gamma T^{(0)^{3}}\right)=\alpha^{*} R^{(0)}\left(T^{(0)^{4}}-T_{f}^{4}\right)+\beta^{*} R^{(0)}\left(T^{(0)}-T_{a}\right),
$$

on $x=1$, where we have set $\alpha=\alpha^{*} / \epsilon$ and $\beta=\beta^{*} / \epsilon$, as this provides the richest limit in which surface radiation and conduction balance convection.

In general, we must solve (30) numerically together with (17). We discretize the differential equations using second-order centred differences for the spatial derivatives, and integrate in time using the MATLAB differentio-algebraic equation solver ode15s, treating (17b) as an algebraic constraint at each time-step. Once the solution is found for an initial guess for $F$, the process is repeated iteratively until boundary condition (13h) is satisfied at the end of the domain.

\subsubsection{Industrially relevant regime}

Based on the parameter values given in Table I, we take $\alpha^{*}=16$, $\beta^{*}=5, \gamma=5$, and consider the effect of the Péclet number, which is directly proportional to the preform width $W_{0}$ and thus the draw ratio, indeed Péclet numbers as high as $O\left(10^{3}\right)$ are possible in highspeed fibre drawing. We present numerical results for the temperature and free-surface profiles for $\mathcal{P}=1,10$ in Fig. 9 (for ease of comparison we have used $z$ rather than the rescaled variable $\zeta$ ). As expected, we see that the temperature now varies across the radius of the fibre, being hottest at the edge and coolest in the middle. Furthermore, this variation is much more pronounced at the larger Péclet number, as suggested from the rescaling (29).

Although we are able to solve the full system numerically, we note that in the limit of strong radiation (as suggested by our parameter values) and/or strong cooling, Eqns. (30a)-(30b) de-couple leading to a simplified system. For example, setting $\alpha=\hat{\alpha} / \epsilon^{2}$ and $\beta=\hat{\beta} / \epsilon^{2}$ where 


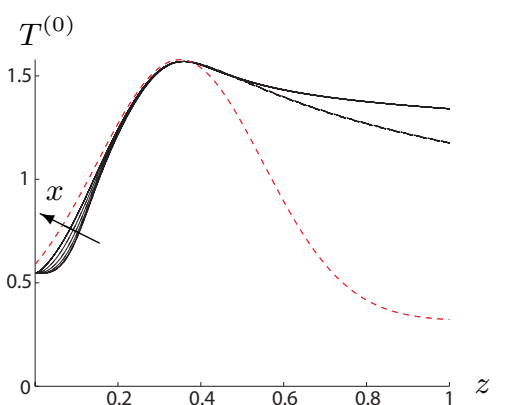

(a)

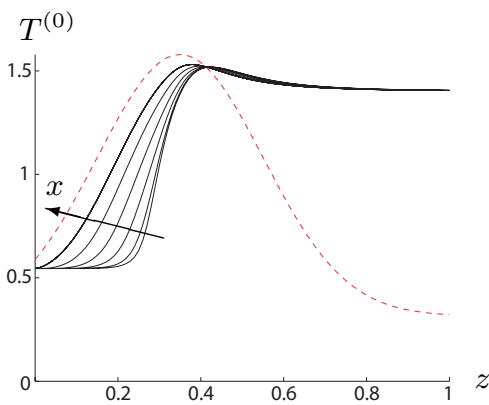

(c)

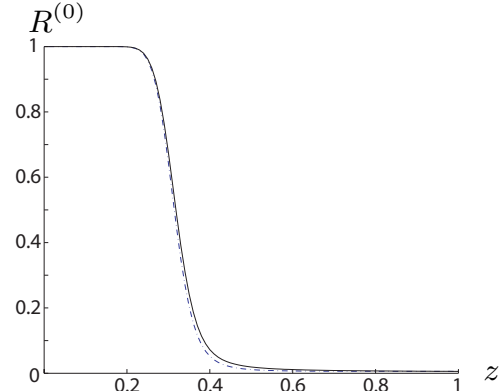

(b)

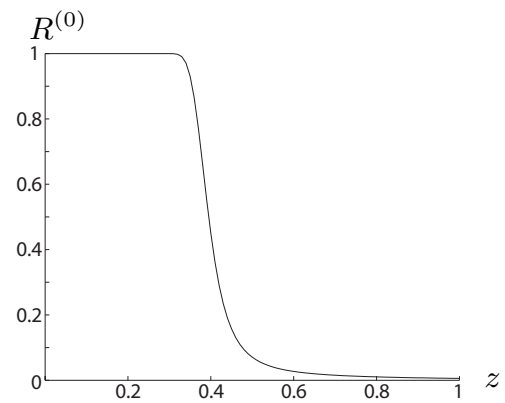

(d)

Figure 9. (a) Variation in leading-order temperature with axial position for radial coordinate $x=\{0,0.2,0.4,0.6,0.8,1\}$ in the case (a) $\mathcal{P}=1$ and (c) $\mathcal{P}=10$; the furnace temperature is also shown (dashed). Figures (b) and (d) show the corresponding fibre radii. In all cases $\alpha=16 / \epsilon, \beta=5 / \epsilon, \gamma=5, w_{1}=3 \times 10^{4}, T_{a}=3 T_{f} / 4$, and $T_{0}=T_{e}$, while in (a),(c) we also show the case of $\alpha(z)$ using (22a) (dot-dashed).

$\hat{\alpha}$ and $\hat{\beta}$ are $O(1)$, the leading-order boundary condition (30b) simplifies to $T^{(0)}=\mathcal{T}(\zeta)$ on $x=1$ where $\mathcal{T}$ is again given by (23) with $\alpha^{*}, \beta^{*}$ replaced by $\hat{\alpha}, \hat{\beta}$ respectively.

\subsubsection{The effect of bulk radiation}

We now consider the role of bulk radiation, which for $P e=O\left(1 / \epsilon^{2}\right)$ comes in at leading order when $\gamma=O(1)$, as assumed thus far. From (30) we see that if $\gamma \gg 1$, bulk radiation dominates so that the temperature is approximately constant across the fibre. On the other hand, if we now set $\gamma=0$, that is, zero bulk radiation, the model reduces to

$$
\begin{aligned}
\frac{\partial T^{(0)}}{\partial \zeta} & =\frac{1}{x} \frac{\partial}{\partial x}\left(x \frac{\partial T^{(0)}}{\partial x}\right) & \text { in } 0 & <x<1, \\
T^{(0)} & =\mathcal{T}(\zeta) & \text { at } x & =1, \\
T^{(0)} & \text { bounded } & \text { at } x & =0, \\
T^{(0)} & =\mathcal{T}(0) & \text { at } \zeta & =0,
\end{aligned}
$$


and in this case we are able to solve explicitly for the temperature. We let $T^{(0)}(x, \zeta)=\mathcal{T}(\zeta)+\hat{T}(x, \zeta)$ and pose the ansatz that

$$
\hat{T}(x, \zeta)=\sum_{m=1}^{\infty} f_{m}(\zeta) J_{0}\left(\lambda_{m} x\right),
$$

where $J_{0}$ is the lowest-order Bessel function and $\lambda_{m}$ are the roots of $J_{0}$. We find that $f_{m}$ satisfies

$$
f_{m}^{\prime}+\lambda_{m}^{2} f_{m}=-\frac{2 \mathcal{T}^{\prime}}{\lambda_{m} J_{1}\left(\lambda_{m}\right)}
$$

where $J_{1}$ is the first-order Bessel function and the solution for $f_{m}$ is given by

$$
f_{m}=-\frac{2 \mathrm{e}^{-\lambda_{m}^{2} \zeta}}{\lambda_{m} J_{1}\left(\lambda_{m}\right)} \int_{0}^{\zeta} \mathcal{T}^{\prime}(s) \mathrm{e}^{-\lambda_{m}^{2} s} \mathrm{~d} s
$$

and so the solution for $T^{(0)}$ is

$$
T^{(0)}(x, \zeta)=\mathcal{T}(\zeta)+\sum_{m=1}^{\infty}-\frac{2 J_{0}\left(\lambda_{m} x\right) \mathrm{e}^{-\lambda_{m}^{2} \zeta}}{\lambda_{m} J_{1}\left(\lambda_{m}\right)} \int_{0}^{\zeta} \mathcal{T}^{\prime}(s) \mathrm{e}^{-\lambda_{m}^{2} s} \mathrm{~d} s .
$$

We show the analytic expression for $T^{(0)}$ given by (35), including ten terms in the summation, in Fig. 10(a) where we see excellent agreement with numerical results everywhere except near the origin, where the modulus of the terms in the expansion start becoming very large, leading to numerical errors. Once we have found the temperature, we may now calculate $\bar{\mu}$ and use (17) to find $w^{(0)}, R^{(0)}$, and $F$. The corresponding fibre radius is shown in Fig. 10(b). In addition to giving a simple explicit solution, (35) also provides a validation for the full two-dimensional numerics.

We compare our solution for zero bulk radiation to one with nonzero bulk radiation, but all other parameters kept constant, in Fig. 10(c)-(d). As expected, with zero bulk radiation we find a greater variation in temperature across the fibre, which leads to a greater radially averaged glass viscosity so that a greater force is required to pull the fibre through the furnace. Increasing $\gamma$ aids the heat transfer through the filament cross-section and hence smooths the temperature out across the fibre. 


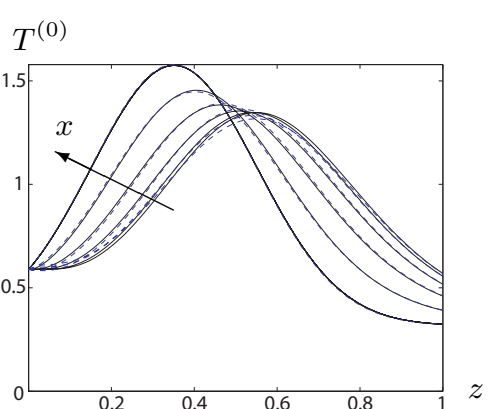

(a)

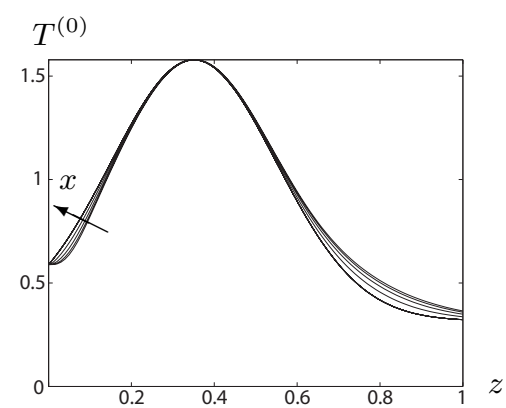

(c)

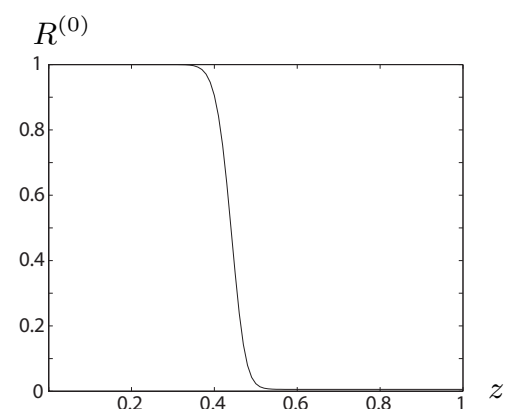

(b)

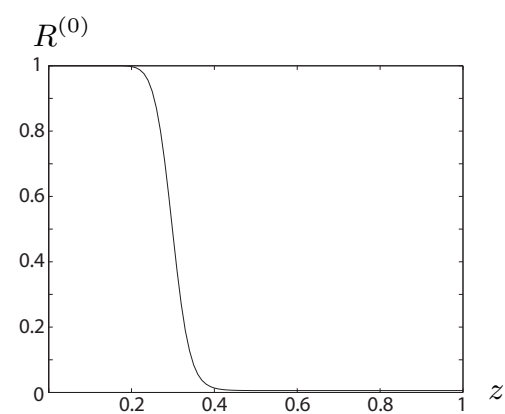

(d)

Figure 10. (a) Variation in leading-order temperature with axial position for $x=\{0,0.2,0.4,0.6,0.8,1\}$ in the case $\mathcal{P}=1, \hat{\alpha}=1, \hat{\beta}=\gamma=0$. The solid lines correspond the numerical solution of $(31 a)$ while the dashed lines correspond to the exact solution (35) taking ten terms in the expansion. The corresponding fibre radius is shown in (b). Figures (c)-(d) are the same as (a)-(b) except $\gamma=5$.

\section{Discussion}

In this paper we have derived and analysed an extensional-flow model to describe the evolution of an optical fibre with axial position drawn at high speed, in which we track the radius of the fibre, the speed of the fibre and the temperature. We incorporated energy transfer due to conduction, convection, bulk and surface radiation, and convective cooling from the air, assuming that the fibre is optically thick. We also included the dependence of the viscosity on temperature.

The model was first solved numerically in the limit where the temperature did not vary across the fibre. Using a change of variables, the solutions were presented in evolving in "time" and exhibited a boundary layer near the end of the evolution. The structure of this solution was determined in the original coordinate system using asymptotic analysis. The domain decomposed into an initial region where the temperature is set by a balance between surface radiation and convective 
cooling, in which the velocity and fibre radius vary significantly, and then a later region where the radius and velocity are effectively constant and the evolution of the temperature also involves bulk convection.

Other interesting parameter limits of the model were explored using asymptotics and numerics. In an industrially relevant limit, the temperature varies along and across the fibre, but the qualitative features are the same as in the radially invariant case. The model can be used to assess the relative importance of surface cooling, surface radiation, bulk radiation and standard heat transfer on the evolution of the fibre. In particular, including bulk radiation smooths the temperature profile across the fibre, as shown in Fig. 10.

Our analysis has always assumed a known (large) pulling speed, with the required force calculated as part of the solution. As expected, this force increases monotonically with the pulling speed, and also increases as the glass viscosity decreases, for example when the Péclet number is particularly high as investigated in $\S 4.2 .2$. In fact, we note that there always exists a force $F$ that allows us to solve our one or twodimensional model, although it may be unfeasibly high for practical purposes. This is in contrast to the more elaborate two and threedimensional models of Jaluria and co-workers, who are unable to find a steady solution if the glass temperature is too low, suggesting that the fibre solidifies and breaks.

Throughout this paper we have restricted ourselves to considering only steady profiles, yet it has long been known that even for isothermal fibre draw ratio the problem becomes unstable once the draw ratio exceeds a critical value $w_{\text {crit }} \approx 20$ (Hyun, 1978). This oscillatory instability is known as draw resonance, and is both of mathematical interest (Renardy, 2006) and great practical importance. A number of authors have thus investigated both the linear (Geyling and Homsy, 1980, Myers, 1989) and nonlinear (Forest and Zhou, 2001) stability of nonisothermal fibre drawing. While it would be possible to follow the same methodology for our model, we note that Scheid et al. (2010) have recently performed an in-depth study on the effect of cooling on fibre drawing, noting that it is governed by the Stanton number $S t=\beta / \epsilon P e$. Provided this is not much greater than unity, as found from our parameter estimates, cooling has a stabilizing effect on fibre drawing, which would aid the process.

As discussed in $\S 2.1$, a limitation in our model is the assumption of an optically thick fibre, which is likely to break down once the fibre is at its thinnest. Unfortunately, determining the energy transfer for an optically thin fibre would involve tracing rays within the fibre; methods to do so have been discussed by, for example, Lee and Jaluria (1997), Lentes and Siedow (1999), and more recently Frank and Klar (2011). 
Secondly, we should solve for the temperature field outside the fibre, taking into account that the convective transfer to the fibre will be affected by the speed of the fibre and that the presence of the air will affect the (external) radiative transfer.

However, the above extensions would necessarily involve computationally intense problems, negating the entire philosophy behind our work, that is, to derive realistic yet simple models for fibre drawing that may be solved quickly. In light of this, useful extensions to our models that could still be readily solved would be the inclusion of multiple layers, as discussed by Cheng and Jaluria (2007), Suman and Tandon (2010), and of viscous dissipation. This latter effect was analysed by Wylie and Huang (2007) for a simplified case, who found that dissipative effects were important once the fibre had thinned, and a one-dimensional analysis required the inclusion of inertial effects. Similarly, Lee and Jaluria (1997) argued that viscous dissipation made two-dimensional effects important, and so it would be expedient to include this effect in future extensions of our work.

\section{Appendix}

\section{A. Phase plane analysis at constant furnace temperature}

As noted in the main text, the system $(18 a, b)$ is amenable to a phase plane analysis in the case where the furnace temperature $T_{f}$ is assumed constant (for consistency with the assumption of $\S 2.1 .4$ the ambient temperature $T_{a}$ is then also constant). Dropping all leading-order (0) superscripts to simplify notation, it proves convenient to consider the phase plane in $(w, \mu)$-space. With $T(\mu)$ given by inverting the relation (14), the autonomous system $(18 \mathrm{a}, \mathrm{b})$ then becomes

$$
\begin{aligned}
& \frac{d w}{d z}=\frac{F w}{3 \mu}, \\
& \frac{d \mu}{d z}=\frac{64 \mu}{P e^{*} T(\mu)^{2} \sqrt{w}}\left(\alpha\left(T(\mu)^{4}-T_{f}^{4}\right)+\beta\left(T(\mu)-T_{a}\right)\right),
\end{aligned}
$$

with $T(\mu)=(1+\log (\mu) / 32)^{-1}$. The phase plane for this system is the solution trajectories of the ODE

$$
\frac{d w}{d \mu}=\frac{P e^{*} F T(\mu)^{2} w^{3 / 2}}{192 \mu^{2}\left(\alpha\left(T(\mu)^{4}-T_{f}^{4}\right)+\beta\left(T(\mu)-T_{a}\right)\right)} .
$$

As in the main text, we can consider either constant $\alpha, \beta$, or $\alpha(w)$, $\beta(w)$ as in, for example, Eqns. (22). Here we present two representative 


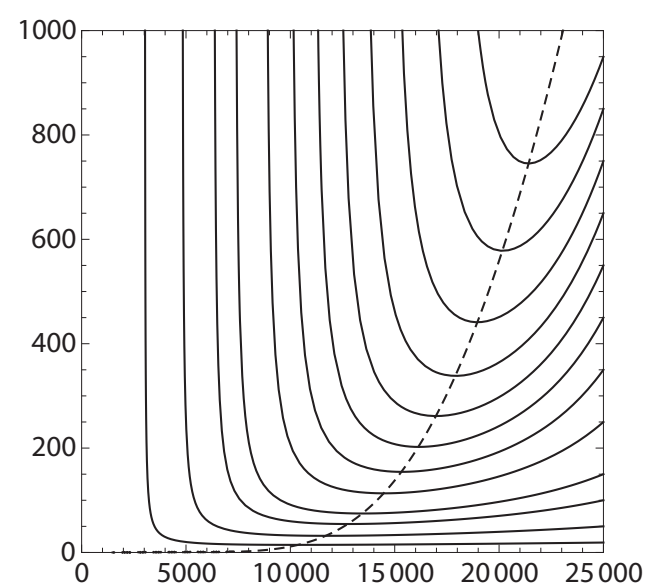

Figure 11. Phase plane for ambient temperature below glass cooling temperature. If the draw ratio is sufficiently high the fibre viscosity may increase again before it exits the furnace.

example phase planes for the case in which $\alpha$ and $\beta$ are functions of $w$. A key factor influencing the qualitative features of the phase diagram is whether the ambient temperature $T_{a}$ is greater than or less than the glass softening temperature. In Fig. 11 we show the phase plane for $\alpha=\alpha_{0}(1-\exp (-10 / \sqrt{w})), \beta=\beta_{0} w^{2 / 3}$, and with $\alpha_{0}=160, \beta_{0}=1$, $T_{f}=1.57, T_{a}=0.75$ (lower than the glass softening temperature, which normalizes to 1 ), and $P e^{*} F=3840$. The solid curves are the phase trajectories, while the dashed curve is the nullcline on which $\alpha\left(T(\mu)^{4}-T_{f}^{4}\right)+\beta\left(T(\mu)-T_{a}\right)=0$. Only a limited range of the $\mu$-axis is shown for simplicity, but it is clear what the evolution would be for such a fibre. The fibre enters at a small value of $w$, the input velocity. At this stage its viscosity is extremely large. As the fibre enters further into the furnace it gains axial velocity and its viscosity drops precipitously, before levelling off. Depending on the draw ratio (ratio of exit velocity to input velocity) the viscosity may reach a minimum value (phase path crossing a nullcline) and then increase again before the fibre exits the furnace. In the case where the ambient temperature $T_{a}$ is higher than the softening temperature the evolution is less dramatic. An example is shown in Fig. 12, with all parameters as before except $T_{a}=1.18>1$. In this case, on entering the furnace the viscosity again drops rapidly on entering the furnace, but then stays at a low value until exit.

Bearing in mind the comments of $\S 4.1 .2$ about the unrealistic nature of the proposed functional dependence of $\beta$, we also show a phase plane for the case of constant $\beta=50$ (Fig. 13). In this case the phase plane shows no qualitative difference between the cases where the ambient temperature is above or below the softening temperature, so 


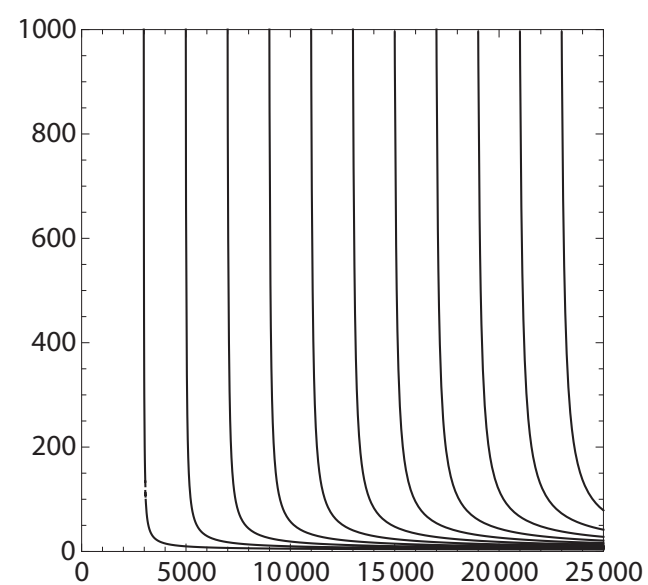

Figure 12. Phase plane for ambient temperature above glass softening temperature. The fibre viscosity decreases monotonically as it passes through the furnace for all draw ratios.

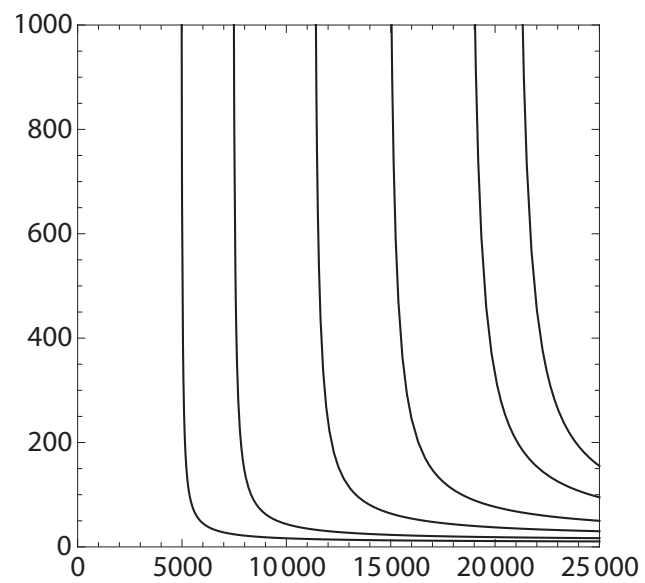

Figure 13. Phase plane for ambient temperature above glass softening temperature, and parameter $\beta$ taken to be constant. The fibre viscosity decreases monotonically as it passes through the furnace for all draw ratios.

we show only the case considered throughout most of the paper, where $T_{a}=3 T_{f} / 4=1.18>1$ (and all other parameters are as above). The fibre viscosity now decreases monotonically as the fibre passes through the furnace, for all draw ratios.

\section{Acknowledgements}

This paper was conceived as an extension to a problem considered at the 27th Annual Workshop on Mathematical Problems in Industry held at 
the New Jersey Institute of Technology in June 2011, with funding from the National Science Foundation. We would like to thank A. Filippov from Corning Inc. who presented the problem at the workshop and gave us invaluable insight into the practical issues involved. We would also like to thank the other participants who worked on different aspects of the problem: R. Beckham, M. Gratton, M. Kanoria, K. Kilgore, V. Lapin, T-S. Lin, M. Ma, H. Nganguia, J. Pohlmeyer, H. Potter, D. Schwendeman, S. K. Wilson, and H. Yaple. Finally, the authors note that this publication was based on work supported in part by Award No KUK-C1-013-04, made by King Abdullah University of Science and Technology (KAUST).

\section{References}

Carslaw, H. S. and J. C. Jaeger: 1959, 'Conduction of heat in solids'. Oxford Clarendon Press, 1959, 2nd Ed.

Cheng, C. and Y. Jaluria: 2007, 'Modeling of radiation heat transfer in the drawing of an optical fiber with multilayer structure'. ASME J. Heat Transfer 129, $342-352$.

Cummings, L. J. and P. D. Howell: 1999, 'On the evolution of non-axisymmetric viscous fibres with surface tension, inertia and gravity'. J. Fluid Mech. 389, 361-389.

Filippov, A.: 2011, '27th Mathematical Problems in Industry workshop'. Private communication.

Fitt, A. D., K. Furusawa, T. M. Monro, C. P. Please, and D. J. Richardson: 2002, 'The mathematical modelling of capillary drawing for holey fibre manufacture'. J. Eng. Math. 43, 201-227.

Forest, G. M. and H. Zhou: 2001, 'Unsteady analyses of thermal glass fibre drawing processes'. Eur. J. Appl. Math. 12, 479-496.

Frank, M. and A. Klar: 2011, 'Radiative Heat Transfer and Applications for Glass Production Processes'. In: Mathematical Models in the Manufacturing of Glass, Lecture Notes in Mathematics. Springer, pp. 57-134.

Geyling, F. T. and G. M. Homsy: 1980, 'Extensional instabilities of the glass fibre drawing process'. Glass Technol. 21, 95-102.

Huang, H., R. M. Miura, and J. J. Wylie: 2008, 'Optical Fiber Drawing and Dopant Transport'. SIAM J. Appl. Math. 69, 330-347.

Hyun, J. C.: 1978, 'Theory of draw resonance: Part I. Newtonian fluids'. AIChE Journal 24, 418-422.

Lee, S. H. K. and Y. Jaluria: 1997, 'Simulation of the transport processes in the neck-down region of a furnace drawn optical fiber'. Int. J. Heat Mass Transfer 40, 843-856.

Lentes, F. T. and N. Siedow: 1999, 'Three-dimensional radiative heat transfer in glass cooling processes'. Glastech. Ber. Glass Sci. Technol. 72, 188-196.

Myers, M. R.: 1989, 'A Model for Unsteady Analysis of Preform Drawing'. AIChE Journal 35, 592-602.

Paek, U. C. and R. B. Runk: 1978, 'Physical behavior of the neck-down region during furnace drawing of silica fibers'. J. Appl. Phys. 49, 4417-4422. 
Papamichael, H. and I. N. Miaoulis: 1991, 'Thermal behavior of optical fibers during the cooling stage of the drawing process'. J. Mater. Res. 6, 159-167.

Renardy, M.: 2006, 'Draw resonance revisited'. SIAM J. Appl. Math. 66, 1261-1269.

Roy Choudhury, S. and Y. Jaluria: 1998, 'Thermal transport due to material and gas flow in a furnace for drawing an optical fiber'. J. Mater. Res. 13, 494-503.

Roy Choudhury, S., Y. Jaluria, and S. H.-K. Lee: 1999, 'A computational method for generating the free-surface neck-down profile for glass flow in optical fiber drawing'. Numer. Heat Transfer Part A 35, 1-24.

Scheid, B., S. Quiligotti, B. Tranh, R. Gy, and H. A. Stone: 2010, 'On the (de)stabilization of draw resonance due to cooling'. J. Fluid Mech. 636, 155-176.

Suman, B. and S. Kumar: 2009, 'Draw ratio enhancement in nonisothermal melt spinning'. AIChE Journal 55, 581-593.

Suman, B. and P. Tandon: 2010, 'Fluid flow stability analysis of multilayer fiber drawing'. Chem. Eng. Sci 65, 5537-5549.

Wylie, J. J. and H. Huang: 2007, 'Extensional flows with viscous heating'. J. Fluid Mech. 571, 359-370.

Wylie, J. J., H. Huang, and R. M. Miura: 2007, 'Thermal instability in drawing viscous threads'. J. Fluid Mech. 570, 1-16.

Yin, Z. and Y. Jaluria: 1997, 'Zonal Method to Model Radiative Transport in an Optical Fiber Drawing Furnace'. ASME J. Heat Transfer 119, 597-603.

Yin, Z. and Y. Jaluria: 2000, 'Neck Down and Thermally Induced Defects in HighSpeed Optical Fiber Drawing'. ASME J. Heat Transfer 122, 351-362. 



\section{RECENT REPORTS}

12/64 A model for one-dimensional morphoelasticity and its application to fibroblast-populated collagen lattices

Menon

Hall

McCue

McElwain

12/65 Effective order strong stability preserving RungeKutta methods

Hadjimichael

Macdonald

Ketcheson

Verner

12/66 Morphoelastic Rods Part I: A Single Growing Elastic Rod

Moulton

Lessinnes

Goriely

12/67 Wrinkling in the deflation of elastic bubbles

Aumaitre

Knoche

Cicuta

Vella

12/68 Indentation of ellipsoidal and cylindrical elastic shells

Vella

Ajdari

Vaziri

Boudaoud

12/69 Memory of Recessions

Cross

McNamara

Pokrovskiif

12/70 An estimate of energy dissipation due to soil-moisture hysteresis

McNamara

12/71 The Mathematics Behind Sherlock Holmes: A Game of Shadows

Goriely

Moulton

12/72 Some observations on weighted GMRES

Güttel

Pestana

12/73 Bounds on the solution of a Cauchy-type problem involving a Furati weighted sequential fractional derivative

12/74 Static and dynamic stability results for a class of threedimensional configurations of Kirchhoff elastic rods

Majumdar

Goriely

12/75 Error estimation and adaptivity for incompressible, nonlinear (hy- Whiteley per)elasticity

Tavener

12/76 A note on heat and mass transfer from a sphere in Stokes flow at low Péclet number

Bell

Byrne

Whiteley

Waters

12/77 Effect of disjoining pressure in a thin film equation with nonuniform forcing

Moulton

Lega

12/78 A Review of Mathematical Models for the Formation of Vascular

Scianna Networks

Bell

Preziosi

12/79 Fast and Accurate Computation of Gauss-Leqendre and Gauss- 
$12 / 80$ On the spectral distribution of kernel matrices related to radial Wathen basis functions

Zhu

12/81 Inner product computation for sparse iterative solvers on dis- Zhu tributed supercomputer

Gu

Liu

12/82 A new pathway for the re-equilibration of micellar surfactant solu-

Griffiths tions

Breward

Colegate

Dellar

Howell

Bain

12/83 Object-Oriented Paradigms for Modelling Vascular Tumour Growth: a Case Study

Connor

Cooper

Byrne

Maini

McKeever

12/84 Chaste: an open source C++ library for computational physiology and biology

Mirams

Arthurs

Bernabeu

Bordas

Cooper

Corrias

Davit

Dunn

Fletcher

Harvey

Marsh

Osborne

Pathmanathan

Pitt-Francis

Southern

Zemzemi

Gavaghan

12/85 A two-pressure model for slightly compressible single phase flow in bi-structured porous media

Schlackow

Marguerat

Proudfoot

Bähler

Erban

Gullerova

12/86 Boolean modelling reveals new regulatory connections between

Lovrics transcription factors orchestrating the development of the ventral spinal cord

Gao

Juhász

Bock

Byrne

Dinnyés

Kovács 
Copies of these, and any other OCCAM reports can be obtained from:

Oxford Centre for Collaborative Applied Mathematics Mathematical Institute

24 - 29 St Giles'

Oxford

OX1 3LB

England

www.maths.ox.ac.uk/occam 